\title{
ODD-SYMPLECTIC FORMS VIA SURGERY AND MINIMALITY IN SYMPLECTIC DYNAMICS
}

\author{
HANSJÖRG GEIGES AND KAI ZEHMISCH
}

\begin{abstract}
We construct an infinite family of odd-symplectic forms (also known as Hamiltonian structures) on the 3 -sphere $S^{3}$ that do not admit a symplectic cobordism to the standard contact structure on $S^{3}$. This answers in the negative a question raised by Joel Fish motivated by the search for minimal characteristic flows.
\end{abstract}

\section{INTRODUCTION}

The plugs for Hamiltonian flows constructed by V. Ginzburg [20, 21] and M. Herman [25, 26] allow one to produce smooth Hamiltonian flows without periodic orbits on compact hypersurfaces in $\mathbb{R}^{2 n}, n \geq 3$. For an alternative construction of Hamiltonian plugs and a guide to the more recent literature on the subject see [15].

The existence of aperiodic Hamiltonian flows prompted Herman at his 1998 ICM address 24 to raise the question whether one can find compact, connected hypersurfaces in $\mathbb{R}^{2 n}, n \geq 2$, on which the characteristic flow is not only aperiodic, but minimal, that is, where every orbit is dense. For $n=2$, this question has recently been answered in the negative by J. Fish and H. Hofer, see [11].

Theorem 1.1 (Fish-Hofer). Let $H$ be a smooth and proper Hamiltonian on $\mathbb{R}^{4}$. Then no energy level of $H$ is minimal.

In this context, Fish has posed a question concerning the existence of certain symplectic cobordisms, very much in the spirit of symplectic dynamics as defined in 3]. Following [19], we call a closed 2 -form $\omega$ on a $(2 n-1)$-dimensional manifold $M$ an odd-symplectic form if $\omega$ is of rank $2 n-2$, that is, if it has a 1-dimensional kernel. The terminology 'Hamiltonian structure' is also in use, see [4]. We shall always assume $M$ to be oriented; equivalently, the characteristic line bundle ker $\omega$ is oriented.

In the following question, the 3 -sphere $S^{3}$ is given its standard orientation as the unit sphere in $\mathbb{R}^{4}$. The contact structure $\xi$ on $S^{3}$ is assumed to be positive, that is, $\alpha \wedge \mathrm{d} \alpha$ is a positive volume form for any choice of contact form $\alpha$ with ker $\alpha=\xi$. Moreover, for condition (ii) below it is assumed that a coorientation and hence orientation for $\xi$ has been chosen by fixing $\alpha$ up to multiplication by a positive function. The symplectic 4 -manifold $(W, \Omega)$ is oriented by the volume form $\Omega \wedge \Omega$, and the boundary $\partial W$ of $W$ is given the induced orientation.

Question 1.2 (Fish). Let $\omega$ be an odd-symplectic form on the 3-sphere $S^{3}=: S_{-}^{3}$. Can one find a contact structure $\xi$ on $S^{3}=: S_{+}^{3}$ and a compact symplectic 4manifold $(W, \Omega)$ such that $\partial W=S_{+}^{3}-S_{-}^{3}$ and

The authors are partially supported by the SFB/TRR 191 'Symplectic Structures in Geometry, Algebra and Dynamics', funded by the Deutsche Forschungsgemeinschaft. 
(i) $\left.\Omega\right|_{T S_{-}^{3}}=\omega$,

(ii) $\left.\Omega\right|_{\xi}>0$ ?

Remark 1.3. With the help of open books or by contact surgeries one can build a symplectic cobordism from $\left(S_{+}^{3}, \xi\right)$ to $\left(S^{3}, \xi_{\text {st }}\right)$, i.e. the 3 -sphere with its standard contact structure, see [10, Theorem 1.2], [16, Theorem 3.4] — this statement hinges on the fact that, as a consequence of Eliashberg's classification of contact structures on the 3 -sphere, $\xi$ is either diffeomorphic to $\xi_{\text {st }}$, or it is an overtwisted contact structure. By concatenating this with a purported cobordism from $\left(S_{-}^{3}, \omega\right)$ to $\left(S_{+}^{3}, \xi\right)$, one would obtain one from $\left(S_{-}^{3}, \omega\right)$ to $\left(S^{3}, \xi_{\text {st }}\right)$. Thus, we may assume that $\left(S_{+}^{3}, \xi\right)=\left(S^{3}, \xi_{\text {st }}\right)$ in Fish's question.

Here is a brief explanation of the relevance of Question 1.2. Given a compact, connected hypersurface $M$ in standard symplectic space $\left(\mathbb{R}^{2 n}, \omega_{\text {st }}\right)$, the pull-back $\omega:=\left.\omega_{\mathrm{st}}\right|_{T M}$ of the symplectic form $\omega_{\mathrm{st}}$ to $M$ is odd-symplectic. The characteristics of $(M, \omega)$ are the integral curves of the line field on $M$ defined by $\operatorname{ker} \omega$. If $M$ is written as the regular level set of a smooth function $H: \mathbb{R}^{2 n} \rightarrow \mathbb{R}$, the characteristics coincide with the flow lines of the Hamiltonian vector field $X_{H}$ defined by $\omega_{\text {st }}\left(X_{H},.\right)=-\mathrm{d} H$, no matter what choice of $H$. Thus, up to parametrisation, the Hamiltonian dynamics is encoded in the odd-symplectic form on the hypersurface.

For a level set $H^{-1}(c)$ as in Theorem 1.1, a cobordism as sought for in Question 1.2 exists: simply enclose $H^{-1}(c)$ inside a large sphere and take $(W, \Omega)$ to be the part of $\left(\mathbb{R}^{4}, \omega_{\mathrm{st}}\right)$ bounded by these two hypersurfaces.

In order to prove Theorem 1.1, Fish and Hofer compactify $\left(\mathbb{R}^{4}, \omega_{\mathrm{st}}\right)$ to the complex projective plane $\mathbb{C P}^{2}$ with its Fubini-Study symplectic form. They then use results of Gromov on the existence of pseudoholomorphic spheres in $\mathbb{C P}^{2}$ to find a nontrivial minimal set of characteristics in $H^{-1}(c)$ as the limit set of ends of what they call feral pseudoholomorphic curves.

For a general odd-symplectic manifold $\left(M^{3}, \omega\right)$, having a cobordism as in Question 1.2 might allow one to carry over parts of this argument. That such a strategy might be viable is exemplified by Hofer's proof [27] of the Weinstein conjecture for overtwisted contact structures. As explained in [16. Corollary 3.5], in this situation the existence of a periodic Reeb orbit (so in particular the non-minimality of the Reeb flow) follows from a study of pseudoholomorphic curves in a symplectic cobordism from the given overtwisted contact 3-manifold to $\left(S^{3}, \xi_{\mathrm{st}}\right)$.

Alas, the main result of this note says that the answer to Question[1.2] is negative, in general.

Theorem 1.4. There is an infinite family of odd-symplectic forms on $S^{3}$, distinguished by a homotopical invariant, for which a cobordism as in Question 1.2 does not exist.

We describe an explicit construction of this infinite family of examples; the homotopical invariant in question and how to compute it will be explained in the process. We shall also discuss the dynamics of these odd-symplectic forms in greater detail.

An earlier result in this direction is due to K. Cieliebak and E. Volkov, see 4, Corollary 6.21]. They exhibit an example of an odd-symplectic form $\omega$ on $S^{3}$ for which there is no topologically trivial symplectic cobordism to an odd-symplectic form on $S^{3}$ whose characteristics are given by the Reeb flow of the standard contact form. The latter condition is not actually more restrictive than what is required by 
Question [1.2, as a construction by Eliashberg [7] allows one to modify the cobordism accordingly, cf. step (iii) in the proof of [12, Theorem 6]. Topological triviality of the cobordism, however, is not assumed in our Theorem 1.4.

The example of Cieliebak and Volkov arises as the boundary of an exotic symplectic ball (containing an exact Lagrangian 2-torus), and the non-existence of the desired cobordism is shown by appealing to Gromov's uniqueness theorem for symplectic structures on $\mathbb{R}^{4}$ standard at infinity. In other words, their example relies on two deep results of 4-dimensional symplectic topology. Our infinite list of examples, by contrast, is constructed by an 'elementary' surgical procedure, and the non-existence of a symplectic cobordism with the described properties follows from McDuff's result [29, cf. [16, Corollary 3.2], which says that if $\left(S^{3}, \xi_{\mathrm{st}}\right)$ arises as one boundary component of a compact symplectic 4-manifold with weakly convex boundary, then the boundary is in fact connected.

\section{IDEA OF THE CONSTRUCTION}

We start with the product manifold $S^{1} \times S^{2}$ and the obvious odd-symplectic form $\omega_{S^{2}}$ obtained by pulling back the standard area form from $S^{2}$ (of area $4 \pi$ ). In a neighbourhood of a circle $S^{1} \times\{*\}$ we can realise $\omega_{S^{2}}$ as an exact form $\mathrm{d} \alpha$ for some contact form $\alpha$. We then perform contact surgery, see [13, Chapter 6], inside this neighbourhood along a Legendrian knot that is topologically isotopic to $S^{1} \times\{*\}$. This surgery, interpreted as a handle attachment, produces a symplectic cobordism (in a sense that we shall specify) from $\left(S^{1} \times S^{2}, \omega_{S^{2}}\right)$ to the surgered odd-symplectic manifold.

In Section 3 we are going to describe this in detail and show that the surgered manifold is a 3 -sphere. The odd-symplectic forms $\omega$ on $S^{3}$ produced in this way (by choosing a Legendrian knot from an infinite family) can be distinguished by the Hopf invariant of the oriented line field $\operatorname{ker} \omega \subset T S^{3}$. We use a version of this invariant due to R. Gompf 22, which can be computed from an almost complex structure $J=J_{\omega}$ on a filling $W_{\omega}$ of $\left(S^{3}, \omega\right)$, that is, a compact manifold with boundary $\partial W_{\omega}=S^{3}$, and with $\omega>0$ on the $J$-invariant tangent 2-plane field $T S^{3} \cap J\left(T S^{3}\right)$. This so-called $d_{3}$-invariant, which has the advantage not to depend on a choice of trivialisation of the tangent bundle $T S^{3}$, will be described in Section 4 and in Section 5 we compute it for our examples.

The non-existence of a symplectic cobordism (as in Question 1.2) from any of these $\left(S^{3}, \omega\right)$ to $\left(S^{3}, \xi_{\text {st }}\right)$ will be shown in Section 6. Assuming there were such a cobordism, we could concatenate it with the surgery cobordism to obtain a cobordism from $\left(S^{1} \times S^{2}, \omega_{S^{2}}\right)$ to $\left(S^{3}, \xi_{\mathrm{st}}\right)$. A result from 17] about symplectic cobordisms between symplectic fibrations would allows us to connect two copies of this cobordism by a symplectic cobordism between the two boundary components $\left(S^{1} \times S^{2}, \omega_{S^{2}}\right)$, or better: by a symplectic cobordism from the empty set to these two manifolds. The resulting manifold would be a connected weak symplectic filling of the disjoint union of two copies of $\left(S^{3}, \xi_{\mathrm{st}}\right)$, contradicting a result of McDuff [29].

Finally, in Section 7 we discuss the dynamics of these examples. After a slight modification of the odd-symplectic form, which does not affect the property of them not being symplectically cobordant to $\left(S^{3}, \xi_{\text {st }}\right)$, the minimality or otherwise of these examples is an open problem.

Remark 2.1. According to a conjecture due to W. Gottschalk, there are no minimal flows on $S^{3}$ whatsoever. From this perspective it is worth noting that our 
construction allows one to produce odd-symplectic manifolds not diffeomorphic to $S^{3}$ for which a symplectic cobordism to $\left(S^{3}, \xi_{\mathrm{st}}\right)$ as in Question 1.2 does not exist. For instance, one can replace $S^{1} \times S^{2}$ in the construction by $S^{1} \times \Sigma_{g}$, with $\Sigma_{g}$ the orientable surface of genus $g$.

There are very few examples of minimal flows on closed 3-manifolds: irrational flows on the 3-torus and on nilmanifolds, and the horocycle flow on the unit tangent bundle of compact hyperbolic surfaces, see [2], [18, Section 2.2], [28, Section 4].

\section{Surgical DESCRIPtion OF THE EXAMPLES}

We begin with the topological aspect of the surgery.

Lemma 3.1. Surgery along $S^{1} \times\{*\} \subset S^{1} \times S^{2}$ with integral framing produces $S^{3}$.

Proof. We split $S^{1} \times S^{2}$ into two solid tori $V_{0}, V_{1}$, with $V_{0}$ a tubular neighbourhood of $S^{1} \times\{*\}$. These solid tori carry canonical longitudes $\lambda_{0}, \lambda_{1}$, respectively, given by (the class of) a fibre in the fibration $S^{1} \times S^{2} \rightarrow S^{2}$. The respective meridians will be denoted by $\mu_{0}, \mu_{1}$. The gluing of $V_{0}, V_{1}$ that gives $S^{1} \times S^{2}$ is described by the identification $\mu_{0}=-\mu_{1}, \lambda_{0}=\lambda_{1}$.

Surgery along $S^{1} \times\{*\}$ with integral framing means that we cut out $V_{0}$ and reglue a solid torus $S^{1} \times D^{2}$ by sending its meridian $\mu:=\{*\} \times \partial D^{2}$ to

$$
k \mu_{0}+\lambda_{0}=-k \mu_{1}+\lambda_{1}
$$

for some $k \in \mathbb{Z}$. Since there is a diffeomorphism of $V_{1}$ that sends $-k \mu_{1}+\lambda_{1}$ to $\lambda_{1}$ (a $k$-fold right-handed Dehn twist along a meridional disc), this gluing amounts to the same as sending $\mu$ to $\lambda_{1}$, which produces $S^{3}$.

Identify the solid torus $V_{0}$ with $S^{1} \times D^{2} \subset S^{1} \times S^{2}$, and write $\theta$ for the $S^{1}$ coordinate. Recall that the Reeb vector field $R$ of a contact form $\alpha$ is defined by the conditions $\mathrm{d} \alpha(R,)=$.0 and $\alpha(R)=1$. We now want to choose a contact form $\alpha_{0}$ on $V_{0}$ with Reeb vector field $R_{0}=\partial_{\theta}$ and $\mathrm{d} \alpha_{0}=\omega_{S^{2}}$. Of course, we could simply set $\alpha_{0}=\mathrm{d} \theta+(x \mathrm{~d} y-y \mathrm{~d} x) / 2$, with $x, y$ cartesian coordinates on $D^{2}$. For the computation of the classical invariants of Legendrian knots inside the contact manifold $\left(V_{0}, \operatorname{ker} \alpha_{0}\right)$, however, it is more convenient to define $\alpha_{0}$ by an identification of $V_{0}$ with a subset of $\left(S^{3}, \alpha_{\mathrm{st}}\right)$, where

$$
\begin{aligned}
\alpha_{\text {st }} & :=\frac{\mathrm{i}}{2}\left(z_{1} \mathrm{~d} \bar{z}_{1}-\bar{z}_{1} \mathrm{~d} z_{1}+z_{2} \mathrm{~d} \bar{z}_{2}-\bar{z}_{2} \mathrm{~d} z_{2}\right) \\
& =r_{1}^{2} \mathrm{~d} \varphi_{1}+r_{2}^{2} \mathrm{~d} \varphi_{2} \\
& =x_{1} \mathrm{~d} y_{1}-y_{1} \mathrm{~d} x_{1}+x_{2} \mathrm{~d} y_{2}-y_{2} \mathrm{~d} x_{2}
\end{aligned}
$$

is the standard contact form on $S^{3} \subset \mathbb{C}^{2}$ with coordinates $z_{j}=r_{j} \mathrm{e}^{\mathrm{i} \varphi_{j}}=x_{j}+\mathrm{i} y_{j}$, $j=1,2$. This is the contact form that defines the standard contact structure $\xi_{\mathrm{st}}=\operatorname{ker} \alpha_{\mathrm{st}}$. Its Reeb vector field is $R_{\mathrm{st}}=\partial_{\varphi_{1}}+\partial_{\varphi_{2}}$.

The vector field $R_{\text {st }}$ defines the Hopf fibration

$$
\begin{aligned}
& \mathbb{C}^{2} \supset S^{3} \longrightarrow S^{2}=\mathbb{C P}^{1} \\
& \left(z_{1}, z_{2}\right) \longmapsto\left[z_{1}: z_{2}\right] \text {. }
\end{aligned}
$$

The 1 -form $\alpha_{\mathrm{st}}$ is a connection form on this principal $S^{1}$-bundle and d $\alpha_{\mathrm{st}}$ is the pull-back of a symplectic form on $S^{2}$ of total area $2 \pi$, cf. the discussion of the Boothby-Wang construction in [13, Section 7.2]. 
Since the total area of the area form $\omega_{S^{2}}$ on $S^{2}$ is $4 \pi$, we may identify $V_{0}=$ $S^{1} \times D^{2}$ with the solid torus

$$
V_{S^{3}}:=\left\{\left(z_{1}, z_{2}\right) \in S^{3}:\left|z_{2}\right| \leq 3 / 4\right\}
$$

in $S^{3}$ such that

(i) $S^{1} \times\{0\}$ is sent to the Reeb orbit

$$
\left\{\left(\mathrm{e}^{\mathrm{i} \theta}, 0\right) \in S^{3}: \theta \in S^{1}=\mathbb{R} / 2 \pi \mathbb{Z}\right\}
$$

of $\alpha_{\mathrm{st}}$

(ii) $\{0\} \times D^{2} \subset S^{1} \times D^{2}$ with area form $\omega_{S^{2}}$ is sent area-preservingly to a transverse disc to the flow of $R_{\mathrm{st}}$ with area form $\mathrm{d} \alpha_{\mathrm{st}}$, and

(iii) the flow lines of $\partial_{\theta}$ are sent to those of $R_{\mathrm{st}}$ (with the parametrisations given by these respective vector fields).

Then define the contact form $\alpha_{0}$ on $V_{0}$ as the pull-back of $\alpha_{\text {st }}$ under this identification. By construction, the vector field $\partial_{\theta}$ is the Reeb vector field of $\alpha_{0}$, so d $\alpha_{0}$ is invariant under the flow of $\partial_{\theta}$, and by (ii) we then have $\mathrm{d} \alpha_{0}=\omega_{S^{2}}$ on $V_{0}$.

We now want to use this identification to describe Legendrian knots in $V_{0}$ topologically isotopic to $S^{1} \times\{0\}$. We call the Reeb orbit in (i) the spine of $V_{S^{3}}$. For the definition of the classical invariants tb (Thurston-Bennequin invariant) and rot (rotation number) of Legendrian knots see [13, Chapter 3].

Lemma 3.2. The closed curve

$$
\gamma: t \longmapsto \frac{1}{\sqrt{2}}\left(\mathrm{e}^{\mathrm{i} t}, \mathrm{e}^{-\mathrm{i} t}\right), \quad t \in S^{1}=\mathbb{R} / 2 \pi \mathbb{Z},
$$

defines an oriented Legendrian knot $L=\gamma\left(S^{1}\right)$ in $\left(V_{S^{3}}, \xi_{\text {st }}\right)$ topologically isotopic in $V_{S^{3}}$ to the spine of that solid torus (and hence a topological unknot in $S^{3}$ ). The classical invariants of this Legendrian unknot, regarded as a knot in $\left(S^{3}, \xi_{\mathrm{st}}\right)$, are $\operatorname{tb}(L)=-1$ and $\operatorname{rot}(L)=0$.

Proof. We have $\dot{\gamma}=\partial_{\varphi_{1}}-\partial_{\varphi_{2}}$, which gives $\alpha_{\mathrm{st}}(\dot{\gamma})=0$, so $L$ is a Legendrian knot. It is a $(1,-1)$-curve on the Hopf torus

$$
\left\{\left|z_{1}\right|=\left|z_{2}\right|=1 / \sqrt{2}\right\} \subset V_{S^{3}},
$$

and hence topologically isotopic to the spine of $V_{S^{3}}$.

The push-off $L^{\prime}$ of $L$ in the Reeb direction is a parallel $(1,-1)$-curve on the Hopf torus, so the linking number of $L$ with $L^{\prime}$ in $S^{3}$, which by definition is the Thurston-Bennequin invariant of $L$, equals -1 .

From $\operatorname{tb}(L)=-1$ and $L \subset S^{3}$ being a topological unknot, one can conclude $\operatorname{rot}(L)=0$ with the help of of the Bennequin inequality [13, Theorem 4.6.36]. Here is a direct proof. We need to verify that along $\gamma$ the vector field $\dot{\gamma}$ does not rotate relative to a global trivialisation of the plane field $\xi_{\mathrm{st}}$. A global trivialisation of the standard contact structure $\xi_{\text {st }}$ on $S^{3}$ is given by the vector field

$$
y_{2} \partial_{x_{1}}+x_{2} \partial_{y_{1}}-y_{1} \partial_{x_{2}}-x_{1} \partial_{y_{2}} .
$$

Along the Legendrian curve

$$
\gamma(t)=\frac{1}{\sqrt{2}}(\cos t, \sin t, \cos t,-\sin t)
$$


this vector field equals

$$
\frac{1}{\sqrt{2}}\left(-\sin t \partial_{x_{1}}+\cos t \partial_{y_{1}}-\sin t \partial_{x_{2}}-\cos t \partial_{y_{2}}\right)
$$

which coincides with the velocity vector field $\dot{\gamma}(t)$. This proves $\operatorname{rot}(L)=0$.

Legendrian unknots in $\left(S^{3}, \xi_{\text {st }}\right)$ have been classified by Eliashberg and Fraser [8], see also [9, Section 3.5] or [14, Theorem 5.1].

Theorem 3.3 (Eliashberg-Fraser). Let $L \subset\left(S^{3}, \xi_{\mathrm{st}}\right)$ be an oriented Legendrian unknot. Then $\operatorname{tb}(L)=n$ with $n$ a negative integer, and $\operatorname{rot}(L)$ lies in the range

$$
\{n+1, n+3, \ldots,-n-3,-n-1\} .
$$

Any such pair of invariants (tb, rot) is realised, and it determines $L$ up to Legendrian isotopy.

These Legendrian unknots are obtained from the one with $t \mathrm{~b}=-1$ and rot $=0$ by stabilisation, which is a local process corresponding to adding zigzags in the front projection. This yields the following result, which implies that any Legendrian unknot in $S^{3}$ can be used, after the identification of $V_{S^{3}} \subset S^{3}$ with $V_{0} \subset S^{1} \times S^{2}$, for the surgery we have in mind.

Proposition 3.4. Any Legendrian unknot in $\left(S^{3}, \xi_{\text {st }}\right)$ can be realised in $V_{S^{3}} \subset S^{3}$ as a knot topologically isotopic in $V_{S^{3}}$ to the spine of that solid torus.

We write $L_{n, r} \subset V_{0}$ for the Legendrian knot corresponding to a Legendrian unknot in $\left(S^{3}, \xi_{\mathrm{st}}\right)$ (as in this proposition) with invariants $\mathrm{tb}=n$ and rot $=r$ in the range allowed by Theorem 3.3 .

We now define $\omega_{n, r}$ as the odd-symplectic form on $S^{3}$ (by Lemma 3.1), obtained by performing contact surgery in the sense of [6, 30] along $L_{n, r} \subset\left(V_{0}, \operatorname{ker} \alpha_{0}\right)$. Here we assume that the surgered-out solid torus is contained in $V_{0}$. Then $\omega_{n, r}$ is defined to coincide with $\omega_{S^{2}}$ away from the surgery region, and with $\mathrm{d} \alpha$ for some extension $\alpha$ of $\alpha_{0}$ over the glued-in solid torus as a contact form defining the contact structure obtained by surgery.

Contact surgery can be interpreted as a symplectic handle attachment, cf. [13, Section 6.2], and then gives rise to a symplectic cobordism from the old to the surgered contact manifold. Here we are only dealing with an odd-symplectic manifold where the odd-symplectic form comes from a contact form near the surgery region, so we have to specify what we mean by 'symplectic cobordism' in this situation.

Definition 3.5. Let $\left(M_{i}, \omega_{i}\right), i=0,1$, be two odd-symplectic manifolds that are closed and of the same dimension. A symplectic cobordism from $\left(M_{0}, \omega_{0}\right)$ to $\left(M_{1}, \omega_{1}\right)$ is a compact symplectic manifold $(W, \Omega)$ with boundary $\partial W=M_{1}-M_{0}$ and such that $\left.\Omega\right|_{T M_{i}}=\omega_{i}, i=0,1$.

Remark 3.6. This is the notion of symplectic cobordism between odd-symplectic manifolds as in [4, Definition 6.1], except that they only consider topologically trivial cobordisms. As Cieliebak and Volkov point out, this notion of symplectic cobordism is not, in general, reflexive (unlike in the following example). This leads them to consider a weaker notion of symplectic cobordism. For our purposes, the definition above is appropriate. In particular, such symplectic cobordisms can be composed by the neighbourhood theorem for hypersurfaces in symplectic manifolds, so the cobordism relation becomes transitive. See also the discussion in [17. 
Write $D_{\rho}^{2} \subset \mathbb{R}^{2}$ for a 2 -disc of radius $\rho$, and $S_{\rho}^{1}=\partial D_{\rho}^{2}$ for the circle of radius $\rho$. For $\rho=1$ we simply write $D^{2}$ and $S^{1}$. Let $\omega_{\mathbb{R}^{2}}$ be the standard symplectic form on $\mathbb{R}^{2}$ or any subset of it. Then the symplectic 4-manifold

$$
\left(W_{0}, \Omega_{0}\right):=\left(\left(D^{2} \backslash \operatorname{Int}\left(D_{1 / 2}^{2}\right)\right) \times S^{2}, \omega_{\mathbb{R}^{2}} \oplus \omega_{S^{2}}\right)
$$

is a symplectic cobordism between two copies of $\left(S^{1} \times S^{2}, \omega_{S^{2}}\right)$.

By attaching a symplectic handle along the outer copy of $\left(S^{1} \times S^{2}, \omega_{S^{2}}\right)$, corresponding to the contact surgery along $L_{n, r}$, we obtain the following result.

Proposition 3.7. There is a symplectic cobordism $\left(W_{n, r}, \Omega_{n, r}\right)$ from $\left(S^{1} \times S^{2}, \omega_{S^{2}}\right)$ to $\left(S^{3}, \omega_{n, r}\right)$.

\section{The $d_{3}$-INVARIANT OF TANGENT 2-PLANE FIELDS}

In order to distinguish the odd-symplectic forms $\omega_{n, r}$ on $S^{3}$ we consider what is essentially the Hopf invariant of the oriented line field ker $\omega_{n, r}$. A normalised section of this line field, together with a trivialisation of the tangent bundle $T S^{3}=S^{3} \times \mathbb{R}^{3}$, determines a map $S^{3} \rightarrow S^{2}$, and the Hopf invariant would be the linking number of the preimages of two regular values.

For our purposes, an invariant introduced by Gompf [22], and now commonly called the $d_{3}$-invariant, is more amenable to computations. It is based on realising a closed 3-manifold with a tangent 2-plane field $\eta$ as the boundary of a compact almost complex 4-manifold such that $\eta$ is the complex line field along the boundary. Since our manifolds come from a construction via symplectic cobordisms, we are in a natural setting for computing the $d_{3}$-invariant. In our application, the plane field $\eta$ will be the the one complementary to $\operatorname{ker} \omega_{n, r}$, with orientation defined by the 2 -form $\omega_{n, r}$.

We first recall the definition of the $d_{3}$-invariant. Thus, let $Y$ be a closed, oriented 3-manifold, and $\eta \subset T Y$ an oriented tangent 2-plane field. Suppose that $Y$ is the boundary of a compact almost complex 4-manifold $(X, J)$ such that $\eta$ equals the complex line field $T Y \cap J(T Y)$. In fact, using obstruction theory, one can show that such an $(X, J)$ exists for any given $(Y, \eta)$, see [22, Lemma 4.4]; in our situation, this will be evident by construction.

When the first Chern class $c_{1}(\eta)$ is a torsion class (hence trivially in the case $\left.Y=S^{3}\right)$, one can make sense of the number $c_{1}^{2}(X, J)$ obtained by squaring the first Chern class of the almost complex structure $J$ on $X$, even though $H^{2}(X ; \mathbb{Z})$ does not have a well-defined intersection pairing. We shall discuss this issue in the actual computations below.

Write $\sigma(X)$ for the signature of $X$, that is, the signature of the intersection pairing on $H_{2}(X ; \mathbb{Z})$. By $\chi(X)$ we denote the Euler characteristic of $X$. The following Theorem is due to Gompf [22, Theorem 4.16]; see also [23, Section 11.3] and [5, Section 2].

Theorem 4.1 (Gompf). For $c_{1}(\eta)$ a torsion class, the rational number

$$
d_{3}(\eta)=\frac{1}{4}\left(c_{1}^{2}(X, J)-3 \sigma(X)-2 \chi(X)\right)
$$

is a homotopy invariant of $\eta$.

This invariant is complete in the following sense: if two tangent 2-plane fields $\eta_{0}, \eta_{1}$ are homotopic over the 2-skeleton of $Y$, and $c_{1}\left(\eta_{0}\right)=c_{1}\left(\eta_{1}\right)$ is a torsion class, then $\eta_{0}$ is homotopic to $\eta_{1}$ if and only if $d_{3}\left(\eta_{0}\right)=d_{3}\left(\eta_{1}\right)$. 
Remark 4.2. For $S^{3}$ the $d_{3}$-invariant takes values in $\mathbb{Z}+1 / 2$. If we take the trivialisation of $T S^{3}$ coming from regarding $S^{3}$ as the unit quaternions, the relation with the Hopf invariant $h$ is given by $d_{3}=-h-1 / 2$. In particular, we have $d_{3}\left(\xi_{\mathrm{st}}\right)=-1 / 2$, which accords with the formula for $d_{3}$ when we regard $\left(S^{3}, \xi_{\mathrm{st}}\right)$ as the boundary of the unit ball in $\mathbb{C}^{2}$.

\section{Computing the $d_{3}$-INVARIANT FOR the EXAmples}

Given an odd-symplectic form $\omega$ on $S^{3}$, we define $d_{3}(\omega)$ as $d_{3}(\eta)$ for an oriented tangent 2-plane field $\eta$ with $\left.\omega\right|_{\eta}>0$. In order to find an almost complex filling of $\left(S^{3}, \omega_{n, r}\right)$ as required for the computation of its $d_{3}$-invariant, we start from $D^{2} \times S^{2}$ with the obvious (almost) complex structure as a filling of $\left(S^{1} \times S^{2}, \omega_{S^{2}}\right)$. Write $\left(X_{n, r}, J_{n, r}\right)$ for the filling of $\left(S^{3}, \omega_{n, r}\right)$ given by the symplectic handle attachment. In statements that do not depend on the specific choice of $n$ and $r$ we simply write $(X, J)$.

5.1. The homology of $X$. The 4-manifold $X$ is a handlebody obtained from a 4-ball by attaching two 2-handles, the first one producing $D^{2} \times S^{2}$, the second one corresponding to the contact surgery. In particular, we have $\chi(X)=3$.

The homology of $X$ is generated by the 2 -spheres

$$
S_{0}^{2}=\{0\} \times S^{2} \subset D^{2} \times S^{2} \subset X
$$

and $S_{1}^{2}$, made up of the core disc of the second handle and a Seifert disc bounded by the Legendrian knot $L=L_{n, r}$ in $D^{2} \times S^{2}$. This Seifert disc is topologically isotopic to $D^{2} \times\{*\}$ in $D^{2} \times S^{2}$ (via an isotopy keeping the boundary of the disc in $S^{1} \times S^{2}$ ).

For the computation of the signature $\sigma(X)$, we need to understand the intersection numbers of the generating 2 -spheres. The 2 -sphere $S_{0}^{2}$ is given the orientation induced by $\omega_{S^{2}}$, i.e. the orientation of the $S^{2}$-factor in $S^{1} \times S^{2}$. The orientation chosen on $S_{1}^{2}$ is the one that coincides with the positive orientation of $D^{2} \times\{*\}$ over the Seifert disc (and with $L$ as the positive boundary of the Seifert disc). We then clearly have

$$
S_{0}^{2} \bullet S_{0}^{2}=0
$$

and

$$
S_{0}^{2} \bullet S_{1}^{2}=1 .
$$

Hence, no matter what the intersection number $S_{1}^{2} \bullet S_{1}^{2}$, the intersection matrix will have signature $\sigma(X)=0$. For the subsequent computations, however, we need to establish the value of this intersection number.

The linking number of any two orbits of the standard Reeb vector field $R_{\mathrm{st}}$ in $S^{3} \subset \mathbb{C}^{2}$ equals 1: the two orbits in either complex coordinate plane make up a positive Hopf link; the other orbits are $(1,1)$-curves on the Hopf tori. Under the identification of $V_{S^{3}}$ with $V_{0}$, the Reeb orbits are sent to curves in the class $S^{1} \times\{*\}$. This means that the 2-discs in $D^{2} \times S^{2}$ bounded by $L$ and its push-off $L^{\prime}$ transverse to the contact structure intersect in one point less than the corresponding discs in $S^{3}$; the latter intersection number is the linking number of $L$ and $L^{\prime}$ in $S^{3}$, which is $\operatorname{tb}(L)$ by definition. The framing for the contact surgery is -1 (i.e. one left twist) relative to the contact framing defined by this push-off $L^{\prime}$, see [13, Example 6.2.7]. In the surgery handle, $L$ bounds the core disc, and its push-off with the extra negative twist a 2-disc parallel to the core disc. It follows that the self-intersection 
number of $S_{1}^{2}$ is accounted for by the intersection of the mentioned discs in $D^{2} \times S^{2}$, hence

$$
S_{1}^{2} \bullet S_{1}^{2}=\operatorname{tb}\left(L_{n, r}\right)-2=n-2,
$$

where tb is computed in $\left(S^{3}, \xi_{\mathrm{st}}\right)$.

So the intersection form on $H_{2}(X ; \mathbb{Z})$ is given by

$$
Q_{n}:=\left(\begin{array}{cc}
0 & 1 \\
1 & n-2
\end{array}\right) \text {. }
$$

5.2. The first Chern class. We now want to compute $c_{1}=c_{1}(X, J)$. To this end, we need to understand the restriction of $T X$ to $S_{0}^{2}$ and $S_{1}^{2}$. Along $S_{0}^{2}$, the tangent bundle $T X$ splits as $T S_{0}^{2} \oplus \mathbb{C}$, hence

$$
\left\langle c_{1},\left[S_{0}^{2}\right]\right\rangle=2 .
$$

For $S_{1}^{2}$, the argument is more involved and analogous to the proof of [22, Proposition 2.3]. The symplectic 2-handle $D^{2} \times D^{2}$ for the contact surgery (which we attach along its lower boundary $\partial D^{2} \times D^{2}$ to $D^{2} \times S^{2}$ ), regarded as an almost complex manifold, is given by $D^{2} \times D^{2} \subset \mathbb{R}^{2} \times \mathbb{i}^{2}$, see [13, Figure 6.4]. Write $q_{1}, q_{2}$ for the cartesian coordinates of the first $\mathbb{R}^{2}$-factor.

Along the attaching circle of the 2-handle, that is,

$$
\partial D^{2} \times\{0\} \subset \partial D^{2} \times D^{2} \subset D^{2} \times D^{2},
$$

we take the complex trivialisation of the tangent bundle of $D^{2} \times D^{2}$ given by the tangent vector $\tau$ to the attaching circle and the outward normal vector $\nu$. This frame differs from the product frame $\partial_{q_{1}}, \partial_{q_{2}}$ by a generator of $\pi_{1}(\mathrm{SO}(2))$. It follows that $\tau, \nu$ extends as a complex frame over $D^{2} \times D^{2}$, since

$$
\mathrm{SO}(2) \subset \mathrm{SU}(2) \subset \mathrm{U}(2),
$$

and $\mathrm{SU}(2)$ is simply connected.

For the following discussion and the notation used see Figure1, which shows the Seifert disc bounded by $L$ in $D^{2} \times S^{2}$ as part of the 2 -sphere $S_{1}^{2}$. Recall that $S_{1}^{2}$ is given the orientation which restricts to the positive orientation of that disc. The second $\mathbb{C}$-factor is regarded as a subset of $S^{2}$ in $D^{2} \times S^{2}$.

The almost complex structure that extends over a symplectic handle corresponding to a contact surgery is the one coming from the symplectisation, i.e. it is one which preserves the contact planes and sends the normal direction to the contact manifold to the Reeb direction (or, up to homotopy, to any direction in the contact manifold transverse to the contact structure).

The $S^{1}$-factor in $V_{0}=S^{1} \times D^{2}$ is transverse to the contact structure that we use to define the surgery, so the contact planes are homotopic (via planes transverse to the $S^{1}$-factor) to planes tangent to the $D^{2}$-factor. Hence, up to homotopy, we may assume that the almost complex structure on $D^{2} \times S^{2} \subset X$ is simply the one corresponding to this product structure, and that the vector field $\tau$ is tangent to the second factor

Lemma 5.1. The vector fields $\nu$ over the 2-handle and $\partial_{x_{1}}$ over the Seifert disc of $L$ in $D^{2} \times S^{2}$ span a complex line bundle $\mathcal{L}_{1}$ over $S_{1}^{2}$ with $c_{1}\left(\mathcal{L}_{1}\right)=1$.

Proof. Along the attaching circle of the 2-handle, which is identified with the Legendrian knot $L$, both $\nu$ and $\partial_{x_{1}}$ lie in the first $\mathbb{C}$-factor, so they span a complex line bundle $\mathcal{L}_{1}$ over all of $S_{1}^{2}$. The frame $\partial_{x_{1}}$ along $L$ extends over the Seifert disc. The 


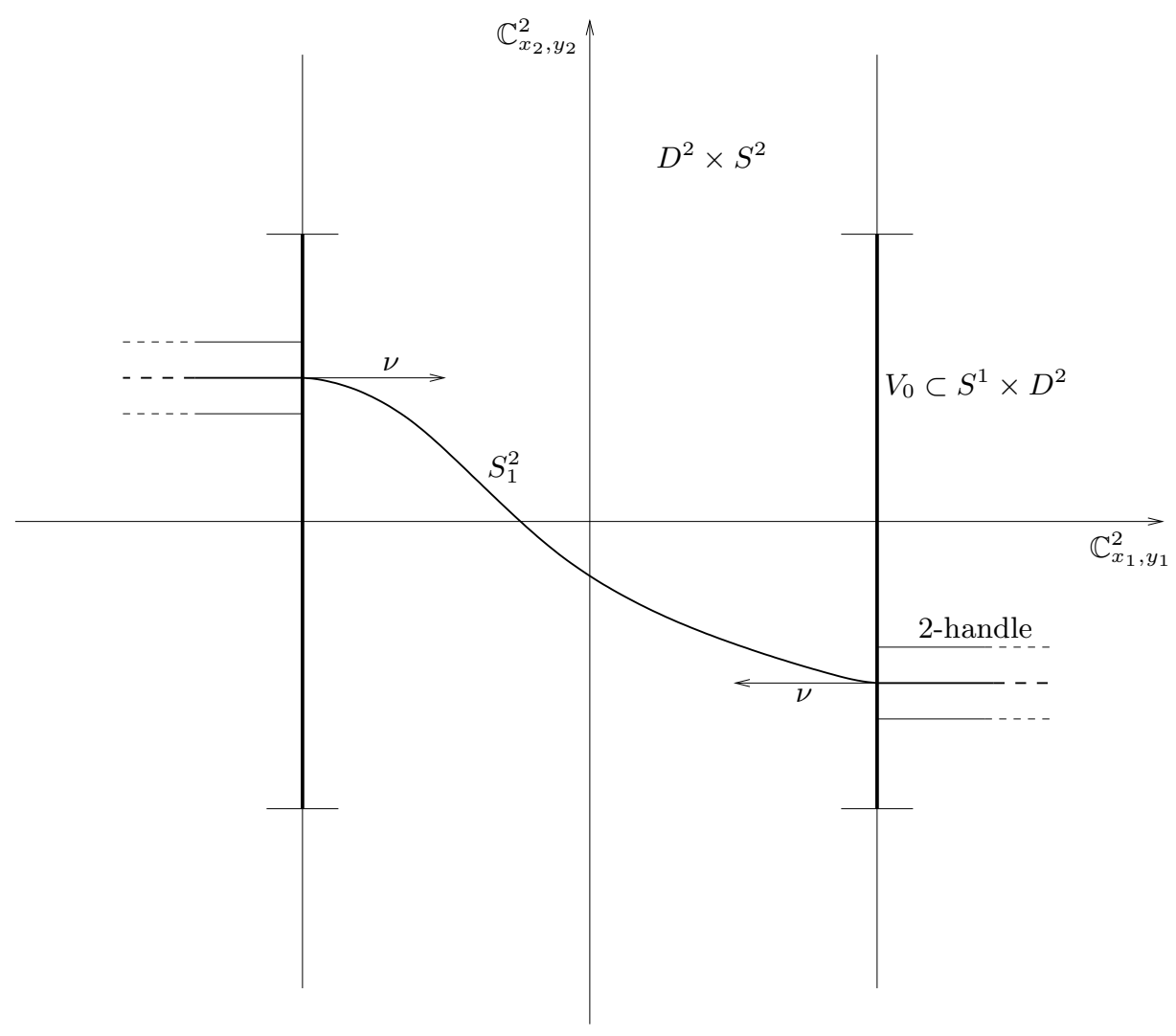

Figure 1. Computing $c_{1}$ from the handle attachment

vector field $\nu$, which is defined over the part of $S_{1}^{2}$ made up by the core disc in the handle, makes one positive twist relative to $\partial_{x_{1}}$ along $L$. From the interpretation of the first Chern class as a relative Euler class it follows that $c_{1}\left(\mathcal{L}_{1}\right)=1$.

Lemma 5.2. The vector fields $\tau$ over the 2 -handle and $\partial_{x_{2}}$ over the Seifert disc of $L=L_{n, r}$ span a complex line bundle $\mathcal{L}_{2}$ with $c_{1}\left(\mathcal{L}_{2}\right)=\operatorname{rot}\left(L_{n, r}\right)-2=r-2$.

Proof. As discussed above, we may think of $\tau$ along $L$ as a vector field in the second $\mathbb{C}$-factor, as is $\partial_{x_{2}}$. So the two vector fields define a complex line bundle $\mathcal{L}_{2}$ over $S_{1}^{2}$.

Along the spine of $V_{S^{3}}$, i.e. the Reeb orbit $\theta \mapsto\left(\mathrm{e}^{\mathrm{i} \theta}, 0\right)$, the vector field in (1) becomes

$$
-\sin \theta \partial_{x_{2}}-\cos \theta \partial_{y_{2}}
$$

Recall that this vector field defines a global trivialisation of $\xi_{\text {st }}$, and the rotation number of an oriented Legendrian knot in $\left(S^{3}, \xi_{\mathrm{st}}\right)$ counts the rotations of the velocity vector relative to this frame. From the expression above we see that this frame makes one left-handed twist relative to the Seifert framing of the spine given by $\partial_{x_{2}}$. In other words, a push-off of the spine in the direction of the global frame of $\xi_{\text {st }}$ has linking number -1 in $S^{3}$ with the spine.

On the other hand, the Reeb orbits of $R_{\mathrm{st}}$, which make up the Hopf fibration, have pairwise linking number +1 . This means that the 'Reeb framing' of $\xi_{\text {st }}$ over 
$V_{S^{3}}$ given by parallel Reeb orbits makes two positive twist relative to the global framing. Hence, the velocity vector field of a oriented Legendrian knot in $V_{S^{3}}$ makes rot -2 rotations relative to the Reeb framing.

In $V_{0} \subset S^{1} \times S^{2}$ the Reeb orbits are of the form $S^{1} \times\{*\}$, so the Reeb framing corresponds to the vector field $\partial_{x_{2}}$. Our discussion shows that $\tau$ makes $r-2$ twists relative to this frame, which implies $c_{1}\left(\mathcal{L}_{2}\right)=r-2$.

Thus, over the sphere $S_{0}^{2}$ the tangent bundle splits as $\mathcal{L}_{1} \oplus \mathcal{L}_{2}$, and the two preceding lemmata imply that $c_{1}(X, J)$ satisfies

$$
\left\langle c_{1},\left[S_{1}^{2}\right]\right\rangle=r-1 .
$$

5.3. Computing $c_{1}^{2}$ and $d_{3}$. The general procedure for computing $c_{1}^{2}(X, J)$ when $c_{1}(X, J)$ restricts to a torsion class on the boundary $\partial X$ is explained in [5, Section 2]. In our situation, where $\partial X=S^{3}$, one can give a more direct argument.

The homology long exact sequence of the pair $\left(X, \partial X=S^{3}\right)$, with integral coefficients understood, gives us an isomorphism

$$
\varphi: H_{2}(X) \longrightarrow H_{2}(X, \partial X)
$$

Given a cohomology class $c \in H^{2}(X)$, there is a unique homology class $C \in H_{2}(X)$ with $\varphi(C)=\mathrm{PD}(c)$, where $\mathrm{PD}: H^{2}(X) \rightarrow H_{2}(X, \partial X)$ is the Poincaré duality isomorphism. Then $c^{2}$ is defined as the intersection number $C^{2}:=C \bullet C \in \mathbb{Z}$.

As mentioned before, $H_{2}(X)$ is generated by the homology classes $\left[S_{0}^{2}\right]$ and $\left[S_{1}^{2}\right]$. The relative homology group $H_{2}(X, \partial X)$ is generated by the classes $\left[N_{0}\right],\left[N_{1}\right]$ of normal discs $N_{0}, N_{1}$ to the knots along which the 2-handles are attached to a 4ball $D^{4}$. Here the handle which gives rise to $N_{0}$ is the one attached along a trivial knot in $D^{4}$ to produce $D^{2} \times S^{2}$, that is, we may take $N_{0}=D^{2} \times\{*\} \subset D^{2} \times S^{2}$, and we choose this disc with $\partial D^{2} \times\{*\}$ disjoint from $V_{0}$, where the second handle is attached. The orientations of $N_{0}, N_{1}$ are chosen such that

$$
N_{0} \bullet S_{0}^{2}=1=N_{1} \bullet S_{1}^{2} \text {. }
$$

In terms of the bases $\left[S_{0}^{2}\right],\left[S_{1}^{2}\right]$ and $\left[N_{0}\right],\left[N_{1}\right]$ for $H_{2}(X)$ and $H_{2}(X, \partial X)$, respectively, the isomorphism $\varphi$ is given by the linking matrix $Q_{n}$ shown in (2).

The Poincaré dual of $c_{1}$ satisfies

$$
c_{1}\left(S_{i}^{2}\right):=\left\langle c_{1},\left[S_{i}^{2}\right]\right\rangle=\mathrm{PD}\left(c_{1}\right) \bullet\left[S_{i}^{2}\right],
$$

which implies that

$$
\mathrm{PD}\left(c_{1}\right)=c_{1}\left(S_{0}^{2}\right) \cdot\left[N_{0}\right]+c_{1}\left(S_{1}^{2}\right) \cdot\left[N_{1}\right]=2\left[N_{0}\right]+(r-1)\left[N_{1}\right] .
$$

Hence, the class $C_{1}=a_{0}\left[S_{0}^{2}\right]+a_{1}\left[S_{1}^{2}\right]$ with $\varphi\left(C_{1}\right)=\operatorname{PD}\left(c_{1}\right)$ is found by solving

$$
\left(\begin{array}{cc}
0 & 1 \\
1 & n-2
\end{array}\right)\left(\begin{array}{l}
a_{0} \\
a_{1}
\end{array}\right)=\left(\begin{array}{c}
2 \\
r-1
\end{array}\right) .
$$

This gives $a_{0}=r-2 n+3$ and $a_{1}=2$. It follows that

$$
c_{1}^{2}=C_{1}^{2}=\left(a_{0}, a_{1}\right)\left(\begin{array}{cc}
0 & 1 \\
1 & n-2
\end{array}\right)\left(\begin{array}{l}
a_{0} \\
a_{1}
\end{array}\right)=(r-2 n+3,2)\left(\begin{array}{c}
2 \\
r-1
\end{array}\right)=4 r-4 n+4
$$

and

$$
\begin{aligned}
d_{3}\left(\omega_{n, r}\right) & =\frac{1}{4}\left(c_{1}^{2}\left(X_{n, r}, J_{n, r}\right)-3 \sigma\left(X_{n, r}\right)-2 \chi\left(X_{n, r}\right)\right) \\
& =r-n-\frac{1}{2} .
\end{aligned}
$$


With Theorem 3.3 we conclude that the values

$$
1 / 2,5 / 2,9 / 2, \ldots
$$

can be realised as $d_{3}$-invariant. This proves the part of Theorem 1.4 saying that we can obtain infinitely many distinct odd-symplectic forms on $S^{3}$ via our surgery construction.

Remark 5.3. Given an odd-symplectic manifold $\left(M^{2 n-1}, \omega\right)$ and a 1 -form $\beta$ on $M$ with $\beta \wedge \omega^{n-1}>0$, one can form the symplectisation $((-\varepsilon, \varepsilon) \times M, \omega+\mathrm{d}(t \beta))$ for $\varepsilon>0$ sufficiently small. Since $\left(S^{3}, \omega_{n, r}\right)$ is distinguished homotopically from $\left(S^{3},\left.\omega_{\mathrm{st}}\right|_{T S^{3}}\right)$, there can be no symplectic embedding of a symplectisation of the former into one of the latter, and no homotopically nontrivial symplectic embedding in the other direction. Any such embedding would give rise to a topologically trivial symplectic cobordism between the two odd-symplectic manifolds, and hence to a homotopy of odd-symplectic forms.

\section{Proof of Theorem 1.4}

Let $\omega=\omega_{n, r}$ be one of the odd-symplectic forms on $S^{3}$ coming from our construction, and let $(W, \Omega)=\left(W_{n, r}, \Omega_{n, r}\right)$ be the corresponding cobordism from $\left(S^{1} \times S^{2}, \omega_{S^{2}}\right)$ to $\left(S^{3}, \omega\right)$ as in Proposition 3.7 .

By [17, Lemma 5], there is a symplectic cobordism from $\left(-S^{1} \times S^{2}, \omega_{S^{2}}\right)$ to $\left(S^{1} \times S^{2}, \omega_{S^{2}}\right)$, which amounts to a cobordism from the empty set to two copies of $\left(S^{1} \times S^{2}, \omega_{S^{2}}\right)$. By gluing a copy of $(W, \Omega)$ along each boundary component we obtain a compact symplectic manifold with two boundary components $\left(S^{3}, \omega\right)$.

Now, assuming there were a symplectic cobordism as in Question 1.2 from $\left(S^{3}, \omega\right)$ to $\left(S^{3}, \xi_{\text {st }}\right)$, we could glue a copy of this cobordism to each boundary of the previously constructed symplectic manifold. The result would be a weak symplectic filling of a disjoint union $\left(S^{3}, \xi_{\mathrm{st}}\right) \sqcup\left(S^{3}, \xi_{\mathrm{st}}\right)$, which cannot exist by [29, Theorem 1.4].

This contradiction proves that the answer to Fish's question is negative for the odd-symplectic forms $\omega_{n, r}$ on $S^{3}$, which completes the proof of Theorem 1.4.

\section{ON THE DYNAMICS OF THE EXAMPLES}

Outside the surgery region, the odd-symplectic forms $\omega_{n, r}$ coincide with $\omega_{S^{2}}$, whose characteristic direction is given by $\partial_{\theta}$. In particular, on $S^{1} \times S^{2} \backslash V_{0}$ (which we may regard as a subset of the surgered $S^{3}$ ), the characteristic flow of $\omega_{n, r}$ is periodic and certainly not minimal.

We now modify $\omega=\omega_{n, r}$ into an odd-symplectic form whose non-minimality is no longer apparent, but which still does not admit a cobordism as requested by Fish. For these examples, then, minimality is an open question.

Write $V \subset V_{0}$ for the solid torus containing $L_{n, r}$ that we cut out when we perform the surgery (equivalently, the image of the attaching map of the 2-handle). Write $\pi: S^{1} \times S^{2} \rightarrow S^{2}$ for the projection onto the $S^{2}$-factor. Define $A:=\pi^{-1}(\pi(V))$; by $\mathcal{O}(A) \subset V_{0}$ we denote a slight thickening of the $S^{1}$-invariant set $A$. Let $\psi: S^{1} \times$ $S^{2} \rightarrow \mathbb{R}$ be a smooth function, independent of the $S^{1}$-coordinate, and let $X_{\psi}$ be the $S^{1}$-invariant vector field which on each slice $\{*\} \times S^{2}$ equals the Hamiltonian vector field of $\psi$, that is,

$$
\omega_{S^{2}}\left(X_{\psi}, .\right)=-\mathrm{d} \psi \text {. }
$$

We may choose $\psi$ in such a way that $\mathrm{d} \psi$ does not have any zeros outside $A$, and such that each level set of $\psi$ (and hence each flow line of $X_{\psi}$ ) meets $A$. 
On $S^{1} \times S^{2}$ we define a 1 -form $\beta_{0}$ by setting it equal to the contact form $\alpha_{0}$ on $A$, equal to $\psi \mathrm{d} \theta$ on $S^{1} \times S^{2} \backslash \mathcal{O}(A)$, and as a convex interpolation on the collar region in between. Then $\beta_{0} \wedge \omega_{S^{2}}>0$ globally on $S^{1} \times S^{2}$. After the surgery, where $\alpha_{0}$ is extended over the glued-in solid torus as a contact form $\alpha$, this extension defines a 1-form $\beta$ with $\beta \wedge \omega$ globally on $S^{3}$, and still $\beta=\psi \mathrm{d} \theta$ on $S^{1} \times S^{2} \backslash \mathcal{O}(A)$, now regarded as a subset of $S^{3}$.

The 2-form

$$
\Omega:=\omega+\mathrm{d}(t \beta)=\omega+\mathrm{d} t \wedge \beta+t \mathrm{~d} \beta
$$

is a symplectic form on $[0, \varepsilon] \times S^{3}$ for $\varepsilon>0$ sufficiently small, and it pulls back to the odd-symplectic forms $\omega$ on $S^{3} \times\{0\}$ and to $\omega_{\varepsilon}:=\omega+\varepsilon \mathrm{d} \beta$ on $S^{3} \times\{\varepsilon\}$, respectively. The odd-symplectic manifold $\left(S^{3}, \omega_{\varepsilon}\right)$ does not admit a cobordism as in Question 1.2, for otherwise we would obtain one for $\left(S^{3}, \omega\right)$ by concatenation.

On $S^{1} \times S^{2} \backslash \mathcal{O}(A) \subset S^{3}$ we have

$$
\omega_{\varepsilon}=\omega+\varepsilon \mathrm{d}(\psi \mathrm{d} \theta)=\omega+\varepsilon \mathrm{d} \psi \wedge \mathrm{d} \theta
$$

The characteristic direction of this odd-symplectic form is given by $\partial_{\theta}-\varepsilon X_{\psi}$. By our choice of $\psi$, this means all characteristics intersect $\mathcal{O}(A)$, and their global dynamics can no longer be controlled.

Acknowledgements. We are grateful to Joel Fish for suggesting this problem about the existence of symplectic cobordisms. We also thank Alberto Abbondandolo, Peter Albers and Ana Rechtman for useful conversations, and Marc Kegel for his critical reading of a draft version of this paper and several constructive comments.

\section{REFERENCES}

[1] L. Auslander, L. Green and F. Hahn, Flows on Homogeneous Spaces, Ann. of Math. Stud. 53, Princeton University Press (1963).

[2] L. Auslander, F. Hahn and L. Markus, Minimal flows on nilmanifolds, Chapter IV of [1].

[3] B. Bramham and H. Hofer, First steps towards a symplectic dynamics, Surv. Differ. Geom. 17, International Press, Boston, MA (2012), 127-177.

[4] K. Cieliebak and E. Volkov, First steps in Hamiltonian topology, J. Eur. Math. Soc. (JEMS) 17 (2015), 321-404.

[5] F. Ding, H. Geiges And A. I. Stipsicz, Surgery diagrams for contact 3-manifolds, Turkish J. Math. 28 (2004), 41-74.

[6] Ya. Eliashberg, Topological characterization of Stein manifolds of dimension $>2$, Internat. J. Math. 1 (1990), 29-46.

[7] Ya. Eliashberg, A few remarks about symplectic filling, Geom. Topol. 8 (2004), 277-293.

[8] Ya. Eliashberg and M. Fraser, Topologically trivial Legendrian knots, J. Symplectic Geom. 7 (2009), 77-127.

[9] J. B. Etnyre and K. Honda, Knots and contact geometry I: Torus knots and the figure eight knot, J. Symplectic Geom. 1 (2001), 63-120.

[10] J. B. Etnyre and K. Honda, On symplectic cobordisms, Math. Ann. 323 (2002), 31-39.

[11] J. W. Fish, Feral pseudoholomorphic curves and minimal sets, Oberwolfach Rep. 12 (2015), 1941.

[12] H. Geiges, Contact Dehn surgery, symplectic fillings, and Property P for knots, Expo. Math. 24 (2006), 273-280.

[13] H. Geiges, An Introduction to Contact Topology, Cambridge Stud. Adv. Math. 109, Cambridge University Press (2008).

[14] H. Geiges and S. Onaran, Legendrian rational unknots in lens spaces, J. Symplectic Geom. 13 (2015), 17-50.

[15] H. Geiges, N. Röttgen and K. Zehmisch, From a Reeb orbit trap to a Hamiltonian plug, Arch. Math. (Basel) 107 (2016), 397-404. 
[16] H. Geiges and K. Zehmisch, How to recognize a 4-ball when you see one, Münster J. Math. 6 (2013), 525-554.

[17] H. Geiges and K. Zehmisch, Cobordisms between symplectic fibrations, Manuscripta Math. 153 (2017), 331-340.

[18] É. Gнys, Dynamique des flots unipotents sur les espaces homogènes, Séminaire Bourbaki 1991/92, Exp. No. 747, Astérisque 206 (1992), 93-136.

[19] V. L. Ginzburg, Calculation of contact and symplectic cobordism groups, Topology 31 (1992), 767-773.

[20] V. L. Ginzburg, An embedding $S^{2 n-1} \rightarrow \mathbb{R}^{2 n}, 2 n-1 \geq 7$, whose Hamiltonian flow has no periodic trajectories, Internat. Math. Res. Notices 1995, 83-97.

[21] V. L. Ginzburg, A smooth counterexample to the Hamiltonian Seifert conjecture in $\mathbb{R}^{6}$, Internat. Math. Res. Notices 1997, 641-650.

[22] R. E. GompF, Handlebody construction of Stein surfaces, Ann. of Math. (2) 148 (1998), 619-693.

[23] R. E. Gompf And A. I. Stipsicz, 4-Manifolds and Kirby Calculus, Grad. Stud. Math. 20, American Mathematical Society, Providence, RI (1999).

[24] M. Herman, Some open problems in dynamical systems, Proceedings of the International Congress of Mathematicians, Vol. II (Berlin, 1998), Doc. Math. (1998), Extra Vol. II, 797808.

[25] M. R. Herman, Examples of compact hypersurfaces in $\mathbb{R}^{2 p}, 2 p \geq 6$, with no periodic orbits, in Hamiltonian systems with three or more degrees of freedom, NATO Adv. Sci. Inst. Ser. C Math. Phys. Sci. 553, Kluwer, Dordrecht (1999), p. 126.

[26] M. R. Herman, Examples of compact hypersurfaces in $\mathbb{R}^{2 p}, 2 p \geq 6$, with no periodic orbits, with comments by F. Laudenbach, available at https://www.college-de-france.fr/ media/jean-christophe-yoccoz/UPL7089_herman_hypersurf.pdf.

[27] H. Hofer, Pseudoholomorphic curves in symplectizations with applications to the Weinstein conjecture in dimension three, Invent. Math. 114 (1993), 515-563.

[28] L. Markus, Lectures in Differentiable Dynamics, American Mathematical Society, Providence, RI (1971).

[29] D. McDuff, Symplectic manifolds with contact type boundaries, Invent. Math. 103 (1991), $651-671$.

[30] A. Weinstein, Contact surgery and symplectic handlebodies, Hokkaido Math. J. 20 (1991), $241-251$.

Mathematisches Institut, Universität zu Köln, Weyertal 86-90, 50931 KÖln, GerMANY

E-mail address: geiges@math.uni-koeln.de

Mathematisches Institut, WWU Münster, Einsteinstrasse 62, 48149 Münster, GerMANY

E-mail address: kai.zehmisch@uni-muenster.de 